\title{
Thrombospondins as matricellular modulators of cell function
}

\author{
Paul Bornstein \\ Departments of Biochemistry and Medicine, University of Washington, Seattle, Washington, USA \\ Address correspondence to: Paul Bornstein, Department of Biochemistry, Box 357350, University of Washington, \\ Seattle, Washington 98195, USA. Phone: (206) 543-1789; Fax: (206) 685-4426; E-mail: bornsten@u.washington.edu.
}

Thrombospondins (TSPs) are a small family of secreted, modular glycoproteins whose functions, at a mechanistic level, are not well understood, despite increasing scrutiny in recent years. TSP1 and TSP2 form one subgroup and are trimers with a chain molecular mass of about $145 \mathrm{kDa}$. The pentameric TSPs 3-5 are significantly smaller, with a subunit molecular mass of about $100 \mathrm{kDa}(1)$, and their physiological roles are probably distinct. This Perspective will concern itself with TSP1 and TSP2.

\section{TSP1 and TSP2 as matricellular proteins}

Unlike the various structural proteins of the ECM, TSP1 and TSP2 do not appear to contribute directly to the integrity of a physical entity, such as a fiber or a basement membrane. Rather, it seems that these proteins act contextually to influence cell function by modulating cell-matrix interactions. TSP1 and TSP2 can interact with specific cell-surface receptors, cytokines, growth factors, and proteases, and the availability of each of these diverse molecules may help define their function in a given environment. These properties are shared by other nonhomologous but functionally similar proteins such as SPARC, tenascin $\mathrm{C}$, and osteopontin, some of which are the subject of other Perspectives in this series, and have led to the application of the term "matricellular" to this group of proteins (2). Because no orthologues of TSP1 and TSP2 can be found in the genomes of Caenorbabditis elegans or Drosophila (3), it appears that these two proteins do not play fundamental roles in metazoan biology but have evolved to deal with the increased complexity of cellmatrix interactions in vertebrates.

Matricellular proteins share a number of other characteristics. These proteins are expressed primarily during development, during growth, and in response to injury, and they are not abundant in the normal adult animal, except in tissues with continued turnover, such as bone. Furthermore, targeted disruption of the murine genes encoding these proteins produces an apparently normal or subtle phenotype, although more careful scrutiny and appropriate challenges have revealed clear-cut and often unanticipated abnormalities (refs. 4-6; and see other Perspectives in this series). These findings are in contrast to the phenotypes of mice with targeted disruptions of genes encoding structural extracellular proteins, such as collagens, laminins, or fibronectin, which are generally severe or lethal. Presumably, the complex regulatory adjustments that are needed for normal development are more likely to occur in the absence of a protein that plays a nonstructural role, since such regulatory pathways are often complex networks with many opportunities for compensatory interactions. However, it should be noted that the distinction between structural and matricellular proteins is not clear-cut. For instance, many structural proteins also function as ligands for cell-surface receptors and activate signaling pathways. Furthermore, recent evidence indicates that proteolytic fragments of structural proteins can play nonstructural roles, e.g., the antiangiogenic function of $\mathrm{COOH}$-terminal sequences in type XVIII collagen (endostatin) and in some type IV collagen chains. Nevertheless, the functions of TSP1 and TSP2 can best be understood within the context of the matricellular concept.

Because TSP1 is an abundant constituent of platelet $\alpha$-granules and is therefore readily purified, an extensive literature exists on the functions of this protein, as revealed by cell culture and other in vitro experiments (7). The antiangiogenic properties of the protein have also been studied in vivo in assays such as the rodent corneal pocket and chick chorioallantoic membrane assays. Although no similarly convenient source of natural TSP2 exists, recombinant TSP2 and a preparation from cultured adrenocortical cells have shown largely similar effects. These in vitro data, however, do not take into account the different developmental and spatial patterns of expression of the two proteins, as judged by in situ hybridization and immunohistochemical analyses $(8,9)$. While there is some overlap, for instance in bone, the age-dependent and tissue-specific patterns of expression of TSP1 and TSP2 are quite different. Indeed, the proximal promoter sequences of the two genes in the mouse are distinct, as are the phenotypes of TSP1 and TSP2 knockout mice $(4,5)$. Furthermore, no detectable compensatory increase of the paralogous gene has been observed in either knockout. Therefore, despite their very similar structures and intrinsic properties, the physiological roles of TSP1 and TSP2 are likely to be different $(7,10)$. 


\section{Matricellular proteins}

E. Helene Sage, Series Editor

\section{Platelet function}

Thrombospondin 1 was first identified as a thrombin-sensitive protein (TSP) that was released in response to activation of platelets by thrombin, hence its name. Upon release from activated platelets, TSP1 binds to the platelet surface in a $\mathrm{Ca}^{2+}$-dependent manner. Platelet-bound TSP1 interacts with integrins $\alpha \operatorname{IIb} \beta 3$ and $\alpha v \beta 3$, with CD36 and the integrin-associated protein (IAP), and with integrin-bound fibrinogen and fibronectin. Early antibody inhibition experiments indicated that TSP1 was required for the secondary secretion-dependent phase of platelet aggregation. More recently, TSP1 was shown to activate $\alpha \operatorname{IIb} \beta 3$ by its binding to IAP (11). This interaction results in spreading of platelets on fibronectincoated surfaces and assembly of a signaling complex containing the integrin, IAP, c-Src, FAK, and Syk protein kinase, and culminates in platelet aggregation. However, these findings must now be reconciled with the report that TSP1-null mice are free of bleeding defects and show normal thrombin-induced platelet aggregation (5). Possibly, compensatory changes in these mice maintain a normal potential for platelet aggregation. TSP1 may also perform other functions in blood, since it is incorporated into fibrin clots and binds to a number of plasma proteins including fib- rinogen, plasminogen, and histidine-rich glycoprotein. On the other hand, platelet TSP1 could function primarily at sites of injury and bleeding to influence macrophages, fibroblasts, and endothelial cells that participate in wound healing.

Paradoxically, although TSP1 is present in platelets and TSP2 is not, it is TSP2-null mice that display a bleeding diathesis (4). Kyriakides et al. (12) have recently reported that megakaryocytes contain abundant TSP2, most of which is probably produced by marrow stromal cells and is taken up by megakaryocytes from the extracellular milieu. Just how TSP2 is lost from megakaryocytes as they fragment to form platelets is not understood. Platelets from TSP2-null animals show a reduced ability to aggregate in vitro in response to ADP and are also compromised in the formation of aggregates on the denuded subendothelium of the common carotid artery of mice in vivo (12). TSP2 therefore seems to be required for the generation of normal platelets from megakaryocytes, but the resulting biochemical defect in TSP2-null platelets is not known. It is also possible, in view of the abnormal collagen fibrillogenesis observed in TSP2-null mice, that abnormalities in the subendothelium could contribute to the defective aggregation of platelets in vivo.

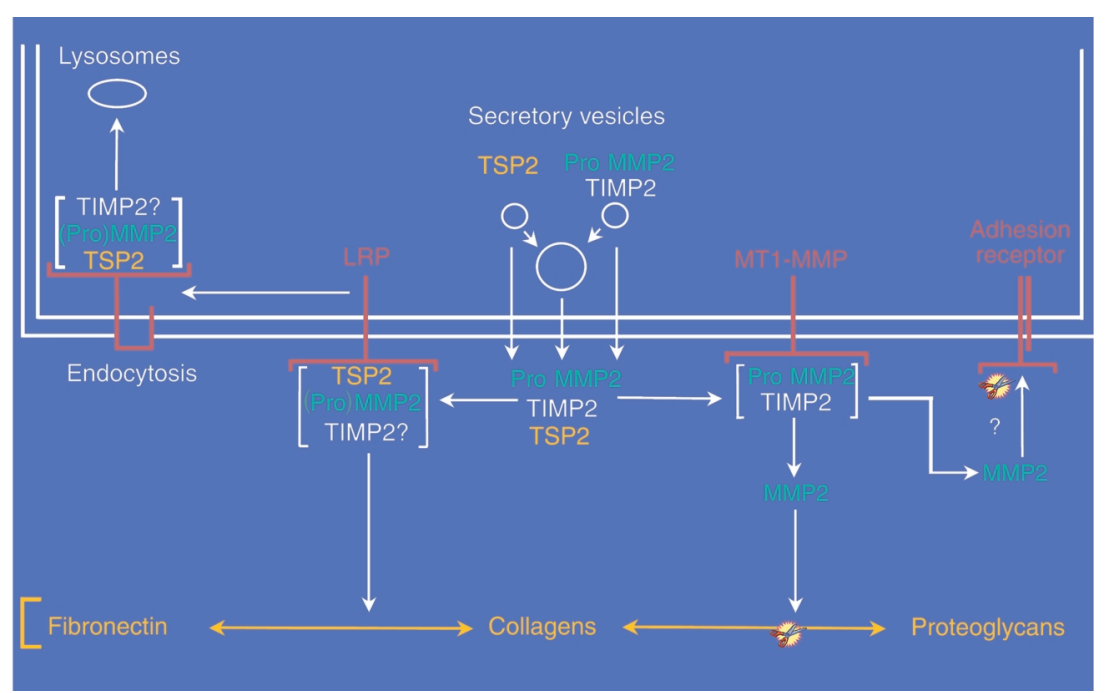

\section{Figure 1}

A proposed scheme to account for the modulation of pericellular MMP2 levels by TSP2. TSP2, ProMMP2, and TIMP2 are secreted into the pericellular environment, either individually or together, by fusion of secretory vesicles with the plasma membrane (double line). As shown on the right side of the figure, ProMMP2, in a complex with TIMP2, can be activated by membrane-bound MT1-MMP to form active MMP2, which is capable of reducing adhesion by cleaving matrix-bound proteins and proteoglycans, and possibly adhesion receptors. When TSP2 is present, a competing pathway clears ProMMP2 from the cell surface. Thus, as shown on the left, TSP2 can bind either ProMMP2 or active MMP2. This complex is then bound by the LRP receptor, endocytosed, and directed to lysosomes for degradation. Since the binding of (Pro)MMP2 to TIMP2 is of considerably higher affinity than that to TSP2, it is possible that a trimolecular complex that includes TIMP2 is endocytosed by LRP. However, TSP2 could still compete effectively with TIMP2 for binding to ProMMP2 or MMP2 by mass action, if the [TSP2] >> [(Pro)MMP2] and if the TSP2/(Pro)MMP2 complex were constantly removed by endocytosis. Alternatively, the TSP2/(Pro)MMP2 complex could be bound to the matrix, thus reducing the bioavailability of the protease. 
Cell-matrix adhesion, motility, and chemotaxis

The ability of TSP1 to serve as an adhesive substratum was documented for a number of tumor and established cell lines (see ref. 13). However, for some normal cells, TSP1 supports attachment but not spreading and is counteradhesive when added to an adhesive protein such as fibronectin (14). TSP1 can reduce focal adhesions in aortic endothelial cells or fibroblasts plated on fibronectin, an activity attributed to heparin-binding sequences in the $\mathrm{NH}_{2}$-terminal globular domain of the protein (15) and mediated by calreticulin and cGMPdependent protein kinase (ref. 16; see also Murphy-Ullrich, this Perspective series, ref. 17). More limited experiments indicated that TSP1 and TSP2 function similarly (15). It was therefore unexpected to find that dermal fibroblasts from TSP2-null mice attached poorly to a number of protein substrata (4). Subsequent experiments showed that the levels of matrix metalloproteinase 2 (MMP2) are twice as high in the conditioned media of TSP2-null fibroblasts as in media of control cells, but that mRNA levels are unchanged (18). These increased MMP2 levels appear to be responsible for the attachment defect, since inhibitors such as tissue inhibitor of metalloproteinases 2 (TIMP2), or a neutralizing antibody to MMP2, can restore normal attachment. Furthermore, restoration of TSP2 synthesis by stable transfection of a TSP2 cDNA gene increases attachment to normal levels and reduces MMP2 levels significantly (18).

How might the absence of TSP2 in TSP2-null fibroblasts lead to increased MMP2 levels in conditioned media? Although both TSP1 and TSP2 inhibit a number of serine proteases (19), TSP2 does not inhibit the gelatinolytic activity of MMP2 $(20,21)$. Since synthesis of the protease does not appear to be increased, it seems possible that clearance of MMP2 from the pericellular environment might be impaired. Indeed, it has been shown recently that TSP2 binds MMP2 directly and that the TSP2/MMP2 complex, which may also include TIMP2, is endocytosed by the LDL receptor-related protein (LRP) and is presumably degraded in lysosomes (21) (Figure 1).

These experiments provide an alternative mechanism to that resulting from cell-surface receptor-mediated interactions for the reduction of adhesion by TSPs. To complicate the picture further, a recent report provides evidence for a proadhesive function of matrix-bound TSP1. Thus, when provided as a substrate for sparsely plated bovine aortic endothelial cells, TSP1 stimulates spreading and proliferation (22). This proangiogenic response has been attributed to interaction of insoluble TSP1 with the $\alpha 3 \beta 1$ integrin.

As might be expected, cell motility and directed migration (both chemotactic and haptotatic) directly reflect the ability of cells to attach to and form transient adhesive interactions with a substratum. Many studies have shown that TSP1 is a potent chemotactic factor for a wide variety of cells, including smooth muscle cells, neutrophils and monocytes, melanoma and squamous carcinoma cells, fibroblasts, and mesangial cells. For smooth muscle cells, at maximally effective concentrations, TSP1 elicits a higher migratory response than either PDGF or bFGF. However, for endothelial cells, the published evidence is not clear-cut; both stimulatory and inhibitory effects have been reported, and these depended on the region of the protein tested and the concentration used $(23,24)$. Certainly, both the type of cell and the substratum influence the adhesive and chemotactic activity of TSPs. Another variable, whose importance is gaining appreciation, is the conformation of these proteins, which may differ depending on whether they are in solution or bound to a matrix. This distinction was probably first made by Taraboletti et al. (25), who observed that different molecular domains mediated haptotaxis and chemotaxis in tumor cells. In all likelihood, these findings reflect the relative availability of domains in the protein to interact with different cell-surface receptors. Heparin-binding sequences and a recognition sequence for $\alpha 3 \beta 1$ integrin in the $\mathrm{NH}_{2}$-terminal domain, the type I repeats, and binding sites for $\alpha v \beta 3$ integrin and IAP in the $\mathrm{COOH}$-terminal domain have been implicated, both positively and negatively, in cell movement. Thus, while it has not been possible to resolve every discrepancy, studies on adhesion and chemotaxis document that TSPs can perform apparently contradictory functions. These functions can best be understood in light of the multiple interactions of matricellular proteins with cell-surface receptors and extracellular effector proteins.

Wound healing, the inflammatory reaction, and TGF- $\beta 1$ Both TSP1 and TSP2 are induced in response to injury. It has therefore been of interest to determine the function of the two proteins in a well-characterized injury model, the healing of a full-thickness excisional skin wound. DiPietro et al. (26), who studied wound healing in the absence or presence of topically applied antisense TSP1 oligonucleotides, found that antisense treatment reduced the number of TSP1-positive macrophages by $50 \%$ or more during the first 3 days of healing and retarded both re-epithelialization and dermal reorganization. However, there was no difference in the total number of mature macrophages in the control and treated wounds. The results were said to be in agreement with studies of TSP1-null mice, but these studies have not been reported in detail.

The marked differences in the consequences, for wound healing, of a lack of TSP 1 and TSP2 were presaged by analyses of the time course of expression and source of the two proteins $(26,27)$. TSP1 mRNA levels are highest 1 day after wounding and fall gradually to almost undetectable levels by day 10 . In contrast, TSP 2 protein levels are first detectable at day 3 and are maximal by day 10 . Activated platelets probably provide the 


\section{Matricellular proteins}

major source of TSP1 during the first 2 days after wounding, after which infiltrating macrophages provide the bulk of this protein; fibroblasts appear to be the major source of TSP2 after wounding.

Excisional wounds in TSP2-null mice are re-epithelialized at the same rate as controls, but scab loss and dermal closure are accelerated in the mutant animals (27). The initial high vascular density of a healing wound persists in the granulation tissue of these mutant animals, which shows increased deposition of fibronectin and an abnormal organization of collagen fibrils. The obverse experiment, in which excisional wounds were examined in transgenic mice that overexpressed TSP1 under control of the keratin 14 promoter, again indicates the intrinsic similarities between the two proteins (28). Thus, scab loss and wound closure are significantly delayed in the transgenic mice, invasion of the wound bed by granulation tissue is retarded, angiogenesis in the healing wound is compromised, and the number of vessels per unit area, the average vessel size, and the total vessel area are all reduced. These findings underscore a number of important points about TSP1 and TSP2. Although the two proteins are structurally similar and appear to have similar functions when introduced artificially, either as the exogenous protein or endogenously by forced expression from a heterologous promoter, their physiological roles can be quite different and reflect synthesis by different cells in different temporal and spatial patterns.

The most prominent features of the TSP1-null mouse are epithelial hyperplasia, leukocytic infiltration, and acute and chronic inflammatory changes involving the lungs (5), as well as milder inflammatory changes in the pancreas (6). These inflammatory responses are unexpected, since as noted above, TSP1 supports neutrophil adhesion and is chemotactic for both neutrophils and monocytes, and TSP1 also participates in the phagocytosis of apoptotic leukocytes by macrophages. One explanation, favored by Crawford et al. (6), holds that, in the absence of TSP1, there is a deficiency in the conversion of latent to active TGF- $\beta 1$. Murphy-Ullrich and Poczatek had previously shown that TSP1 can activate latent TGF- $\beta 1$ in vitro (29). Activation of latent TGF- $\beta 1$ requires a WXXW binding sequence present in each of the three type I repeats of TSP1, as well as a KRFK activating sequence between the first and second repeats. A deficiency of TGF- $\beta 1$ could lead to an exaggerated immune inflammatory response to normal pulmonary bacterial flora (7), and indeed, TSP1-null mice show many of the histological features of TGF- $\beta 1$-null mice, albeit in milder form. Moreover, young TSP1-null mice treated with a KRFK peptide show a partial reversion of these phenotypes $(6,29)$. If this peptide helps restore normal tissue levels of TGF- $\beta 1$, it is unclear why the WXXW binding sequence is not required in vivo as it is in vitro.

The physiological relevance of the ability of TSP1 to activate latent TGF- $\beta 1$ has been the subject of much attention and some controversy (29). Other means of activation exist, including limited proteolysis by plasmin and other enzymes and interaction with $\alpha v \beta 6$ integrin. In inflammatory conditions, such as bleomycininduced pulmonary fibrosis, CD36 is required together with TSP1. Although the WXXW motifs are conserved in TSP2, the activating KRFK sequence is not. MurphyUllrich and Poczatek suggest that TSP2 could therefore compete with TSP1 for binding to latent TGF- $\beta 1$ and thus inhibit its activation (29). Some of the changes observed in wound healing in TSP2-null mice are indeed compatible with the presence of higher levels of active TGF- $\beta 1$ in the wounds. My laboratory has tested this suggestion by measuring active TGF- $\beta 1$ in cultured dermal TSP2-null fibroblasts, which produce TSP1 and latent TGF- $\beta 1$, and we have found that levels of active TGF- $\beta 1$ are, surprisingly, lower than those in control cells. We obtained similar results by comparing active TGF- $\beta 1$ levels in subdermally implanted polyvinyl alcohol sponges from TSP2-null and control mice. Nevertheless, the available evidence supports a physiological role for TSP1 in the activation of latent TGF- $\beta 1$ under some circumstances and, more generally, supports the proposal that the functions of matricellular proteins derive in large part from their interactions with effector proteins.

\section{Angioinhibitory and antitumor activities}

The first indication that TSPs possesses both antiangiogenic and antitumor activities came from the identification of the protein encoded by a tumor suppressor gene as a fragment of TSP1 (30). Subsequent studies were aimed at the identification of peptide sequences, primarily in TSP1, that could be implicated in the inhibition of angiogenesis, the cell-surface receptors with which these sequences interacted, and more recently a delineation of consequent downstream events. These
Table 1

Proposed mechanisms for the angioinhibitory effects of TSPs

\begin{tabular}{|c|c|c|c|}
\hline $\begin{array}{l}\text { Amino acid } \\
\text { sequence }\end{array}$ & Domain & $\begin{array}{l}\text { Cell-surface receptor and } \\
\text { proposed cellular effects }\end{array}$ & Reference \\
\hline NVR & $\mathrm{NH}_{2}$-terminal & $\begin{array}{c}\alpha 3 \beta 1 \text {; inhibition of EC } \\
\text { proliferation }\end{array}$ & (22) \\
\hline CSVTCG & Type I repeats & CD36; apoptosis & $(33,35)$ \\
\hline$w x x w x x w$ & Type I repeats & $\begin{array}{l}\text { HSPG; inhibition of } \\
\text { bFGF binding }\end{array}$ & $(34)$ \\
\hline RFYVMWK & $\mathrm{COOH}$-terminal & $\begin{array}{c}\text { IAP; tyrosine } \\
\text { phosphorylation of FAK }\end{array}$ & (36) \\
\hline
\end{tabular}

HSPG, heparan sulfate proteoglycan. 
studies evaluated the ability of TSPs and TSP-derived fragments or peptides to modify a number of cellular responses, including the angiogenic response to bFGF or VEGF in the rodent corneal pocket or chick chorioallantoic membrane assays, the innate tendency of endothelial cells to form cellular cords or tubes in vitro, and the proliferation, chemotaxis, and migration of endothelial cells (31-33). The results of some of these experiments are summarized in Table 1. TSP1 and TSP2 could function as angioinhibitory proteins by binding bFGF and inhibiting its interaction with syndecans and FGF receptors; by otherwise interfering with bFGFinduced signaling (34); by interaction with CD36, leading to increased caspase-3 activity and apoptosis (35); or by signaling via integrin and IAP receptors. There is also evidence that interaction between IAP and the $\mathrm{COOH}$ terminal peptide (RFYVVMWK, present in both TSP1 and TSP2) inhibits tube formation by endothelial cells and blocks integrin-dependent tyrosine phosphorylation of focal adhesion kinase (36). Finally, it is possible that the organization of the collagenous matrix and MMP2 levels could participate in the regulation of angiogenesis, since disordered collagen fibrils and increased MMP2 levels are observed in the TSP2 knockout mouse and TSP2-null fibroblasts, respectively (4, 18). At this time, CD36-mediated mechanisms are the best documented (35).

Perhaps the most definitive evidence for the antiangiogenic properties of TSP1 and TSP2 comes from in vivo experiments. Thus, both tumor growth and angiogenesis are inhibited in transgenic mice that overexpress TSP1 (37), and nude mice injected with TSP1 or TSP2 cDNA-transfected human squamous carcinoma cells show markedly reduced tumor growth compared with mice injected with vector-transfected cells (38). In these experiments, it appears that the proliferation of tumor cells was not affected by expression of TSP 1 or 2 but that tumor angiogenesis was greatly inhibited. Nevertheless, over the past 5 or 6 years occasional reports have appeared supporting a proangiogenic function for TSP1. Chandrasekaran et al. (22) may have provided a partial explanation for this controversy. These investigators have shown that engagement of the $\alpha 3 \beta 1$ integrin by a soluble TSP1 peptide inhibits endothelial cell proliferation and angiogenesis, whereas the same peptide stimulates endothelial cell proliferation when immobilized. Interpretation of results was more complex when larger TSP1 fragments or the intact protein was used, but these data also emphasize the importance of protein conformation as a determinant of TSP function.

In view of the widely acknowledged dependence of tumor growth and metastasis on angiogenesis, it is reasonable to conclude that many of the recognized inhibitory effects of TSP1 stem from its ability to inhibit endothelial cell proliferation, induce apoptosis, or otherwise interfere with capillary formation (33). However, the tumor stroma, composed predom- inantly of fibroblasts and their matrix, clearly plays a role in tumor growth, and this environment is also susceptible to the effects of the TSPs. Finally, tumor cells themselves can be modulated by TSPs. Such effects include regulation of attachment, cell-cell adhesion, migration, and proliferation. Some of these effects could result from activation of latent TGF- $\beta 1$ or from association between the TSPs and secreted proteases, cytokines, and other effector proteins. Thus, tumors and their adjacent host tissues can be considered microcosms of the organism, and the complex functions of TSPs appear to apply equally to both systems.

\section{Conclusions and prospects for future research}

Although TSPs and their functional relatives are residents of the extracellular space, it is helpful and appropriate to think of these proteins as performing regulatory functions. Such functions can be achieved by interactions with cell-surface receptors and the consequent activation of signaling pathways, by altering the activity of cytokines and growth factors that serve as signaling molecules, or by affecting the protease balance in the pericellular space. All of these effects have now been documented to some extent in vitro. The challenge for the future is to assess which of these mechanisms operate in the more complex environment of the whole animal and to assign specific functions to the various domains of these modular proteins. Mice that lack one or more of these proteins are attractive test beds for such studies. The generation of knock-in mice expressing truncated or mutated proteins represents another potentially informative approach to these questions. Another major question, which might be approached initially by experiments in vitro, is the extent to which the complex functions of matricellular proteins are achieved in an autocrine fashion or require the cooperation of neighboring, probably different, cell types. The latter possibility is not unlikely when one considers the complexity of tissue organization in higher animals.

\section{Acknowledgments}

This work was supported by NIH grants AR-45418 and HL-18645, and by National Science Foundation grant ECC 9529161. I thank members of my laboratory for helpful discussions and comments on the manuscript and regret the need to omit many appropriate references.

\footnotetext{
1. Bornstein, P., and Sage, E.H. 1994. Thrombospondins. Methods Enzymol. 245:62-85.

2. Bornstein, P. 1995. Diversity of function is inherent in matricellular proteins: an appraisal of thrombospondin 1. J. Cell Biol. 130:503-506.

3. Hynes, R.O., and Zhao, Q. 2000. The evolution of cell adhesion. J. Cell Biol. 150:F89-F95.

4. Kyriakides, T.R., et al. 1998. Mice that lack thrombospondin 2 display connective tissue abnormalities that are associated with disordered collagen fibrillogenesis, an increased vascular density, and a bleeding diathesis. J. Cell Biol. 140:419-430.

5. Lawler, J., et al. 1998. Thrombospondin-1 is required for normal murine pulmonary homeostasis and its absence causes pneumonia. J. Clin. Invest.
} 
101:982-992.

6. Crawford, S.E., et al. 1998. Thrombospondin-1 is a major activator of TGF- $\beta 1$ in vivo. Cell. 93:1159-1170.

7. Lawler, J. 2000. The functions of thrombospondin-1 and -2. Curr. Opin. Cell Biol. 12:634-640.

8. Iruela-Arispe, M.L., Liska, D.J., Sage, E.H., and Bornstein, P. 1993. Differential expression of thrombospondin 1,2, and 3 during murine development. Dev. Dyn. 197:40-56.

9. Tooney, P.A., Sakai, T., Sakai, K., Aeschlimann, D., and Mosher, D.F. 1998. Restricted localization of thrombospondin-2 protein during mouse embryogenesis: a comparison to thrombospondin-1. Matrix Biol. 17:131-143.

10. Bornstein, P., Armstrong, L.C., Hankenson, K.D., Kyriakides, T.R., and Yang, Z. 2000. Thrombospondin 2, a matricellular protein with diverse functions. Matrix Biol. 19:557-568.

11. Chung, J., Gao, A.-G., and Frazier, W.A. 1997. Thrombospondin acts via integrin-associated protein to activate the platelet integrin $\alpha I I b \beta 3$. J. Biol. Chem. 272:14740-14746.

12. Kyriakides, T.R., et al. 2000. Lack of uptake of TSP2 by megakaryocytes in TSP2-null mice: a potential explanation for the bleeding diathesis and platelet abnormality. Blood. 96:815a. (Abstr.)

13. Murphy-Ullrich, J.E., and Höök, M. 1989. Thrombospondin modulates focal adhesions in endothelial cells. J. Cell Biol. 109:1309-1319.

14. Sage, E.H., and Bornstein, P. 1991. Extracellular proteins that modulate cell-matrix interactions: SPARC, tenascin and thrombospondin. J. Biol. Chem. 266:14831-14834.

15. Murphy-Ullrich, J.E., Gurusiddappa, S., Frazier, W.A., and Höök, M. 1993. Heparin-binding peptides from thrombospondins 1 and 2 contain focal adhesion-labilizing activity. J. Biol. Chem. 268:26784-26789.

16. Goicoechea, S., et al. 2000. Thrombospondin mediates focal adhesion disassembly through interactions with cell surface calreticulin. J. Biol. Chem. 275:36358-36368.

17. Murphy-Ullrich, J.E. 2001. The de-adhesive activity of matricellular proteins: is intermediate cell adhesion an adaptive state? J. Clin. Invest. 107:785-790.

18. Yang, Z., Kyriakides, T.R., and Bornstein, P. 2000. Matricellular proteins as modulators of cell-matrix interactions: the adhesive defect in thrombospondin 2-null fibroblasts is a consequence of increased levels of matrix metalloproteinase-2. Mol. Biol. Cell. 11:3353-3364.

19. Hogg, P.J. 1994. Thrombospondin 1 as an enzyme inhibitor. Thromb. Haemost. 72:787-792.

20. Bein, K., and Simons, M. 2000. Thrombospondin type 1 repeats interact with matrix metalloproteinase 2: regulation of metalloproteinase activity. J. Biol. Chem. 275:32167-32173.

21. Yang, Z., Strickland, D.K., and Bornstein, P. 2001. Extracellular MMP2 levels are regulated by the LRP scavenger receptor and thrombospondin 2. J. Biol. Chem. 276:8403-8408.

22. Chandrasekaran, L., et al. 2000. Cell contact-dependent activation of $\alpha 3 \beta 1$ integrin modulates endothelial cell responses to thrombospondin1. Mol. Biol. Cell. 11:2885-2900.

23. Taraboletti, G., Roberts, D., Liotta, L.A., and Giavazzi, R. 1990. Platelet thrombospondin modulates endothelial cell adhesion, motility, and growth: a potential angiogenesis regulatory factor. J. Cell Biol. 111:765-772.

24. Tolsma, S.S., et al. 1993. Peptides derived from two separate domains of the matrix protein thrombospondin-1 have anti-angiogenic activity. $J$. Cell Biol. 122:497-511.

25. Taraboletti, G., Roberts, D.D., and Liotta, L.A. 1987. Thrombospondininduced tumor cell migration: haptotaxis and chemotaxis are mediated by different molecular domains. J. Cell Biol. 105:2409-2415.

26. DiPietro, L.A., et al. 1996. Thrombospondin 1 synthesis and function in wound repair. Am. J. Pathol. 148:1851-1860.

27. Kyriakides, T.R., Tam, J.W.Y., and Bornstein, P. 1999. Accelerated wound healing in mice with a disruption of the thrombospondin 2 gene. $J$. Invest. Dermatol. 113:782-787.

28. Streit, M., et al. 2000. Thrombospondin-1 suppresses wound healing and granulation tissue formation in the skin of transgenic mice. EMBO J. 19:3272-3282.

29. Murphy-Ullrich, J.E., and Poczatek, M. 2000. Activation of latent TGF$\beta$ by thrombospondin-1: mechanisms and physiology. Cytokine Growth Factor Rev. 11:59-69.

30. Good, D.J., et al. 1990. A tumor suppressor-dependent inhibitor of angiogenesis is immunologically and functionally indistinguishable from a fragment of thrombospondin. Proc. Natl. Acad. Sci. USA. 87:6624-6628.

31. DiPietro, L.A. 1997. Thrombospondin as a regulator of angiogenesis. In Regulation of angiogenesis. L.D. Goldberg and E.M. Rosen, editors. Birkhauser Verlag. Basel, Switzerland. 295-314.

32. Sheibani, N., and Frazier, W.A. 1999. Thrombospondin-1, PECAM-1 and regulation of angiogenesis. Histol. Histopathol. 14:285-294.

33. Dawson, D.W., and Bouck, N.P. 1999. Thrombospondin as an inhibitor of angiogenesis. In Antiangiogenic agents in cancer therapy. B.A. Teicher, editor. Humana Press. Totowa, New Jersey, USA. 185-203.

34. Iruela-Arispe, M.L., Lombardo, M., Krutzsch, H.C., Lawler, J., and Roberts, D.D. 1999. Inhibition of angiogenesis by thrombospondin-1 is mediated by 2 independent regions within the type 1 repeats. Circulation. 100:1423-1431

35. Jiménez, B., et al. 2000. Signals leading to apoptosis-dependent inhibition of neovascularization by thrombospondin-1. Nat. Med. 6:41-48.

36. Kanda, S., Shono, T., Tomasini-Johansson, B., Klint, P., and Saito, Y. 1999. Role of thrombospondin-1-derived peptide, 4N1K, in FGF-2induced angiogenesis. Exp. Cell Res. 252:262-272.

37. Streit, M., et al. 1999. Overexpression of thrombospondin-1 decreases angiogenesis and inhibits the growth of human cutaneous squamous cell carcinomas. Am. J. Pathol. 155:441-452.

38. Streit, M., et al. 1999. Thrombospondin-2: a potent endogenous inhibitor of tumor growth and angiogenesis. Proc. Natl. Acad. Sci. USA. 96:14888-14893. 